\title{
Persistent hyperglycemia modulates gut immune function and microbiota in rats
}

\author{
Katsuya Mori ${ }^{1}$, Takeshi Suzuki ${ }^{*}$, Toru Igarashi ${ }^{1}$, Kei Inoue ${ }^{1}$, Takashi Asahara ${ }^{2}$, Koji Nomoto $^{2}$, Hiroyuki Seki ${ }^{1}$, \\ Takashige Yamada ${ }^{1}$, Shizuka Minamishima' ${ }^{1}$, Shizuko Kosugi ${ }^{1}$, Nobuyuki Katori ${ }^{1}$ and Hiroshi Morisaki ${ }^{1}$
}

\begin{abstract}
Background: Since hyperglycemia-induced cellular dysfunction could be associated with alterations of the immune system, we tested the hypothesis that hyperglycemia augments the aberrant immune responses such as inflammation and differentiation of $\mathrm{CD}^{+} \mathrm{T}$ lymphocytes in the mesenteric lymph nodes (MLNs), and induces alterations of microbiota both under physiological and pathological conditions.

Methods: Male Wistar rats were randomly allocated into 4 groups: Control and Endotoxemia (lipopolysaccharide, LPS $1 \mathrm{mg} / \mathrm{kg}$ ) with or without hyperglycemia. The hyperglycemia groups were administered glucose solution (10-40\%), while the normoglycemia groups were administered saline. Alterations of the mRNA expressions of inflammatory cytokines and $\mathrm{CD}^{+} \mathrm{T}$ lymphocyte transcriptional factor expressions in the MLNs, and those of the intestinal microbiota were analyzed at $24 \mathrm{hr}$.
\end{abstract}

Results: Hyperglycemia was kept approximately 250-350 mg/dL during the $24 \mathrm{hr}$ study period. At the end of $24 \mathrm{hr}$, hyperglycemia augmented the mRNA expressions of interleukin (IL)-1 $\beta$ and IL- 6 in the MLNs, while both the helper $\mathrm{T}$ (Th) 2 and regulatory-T (Treg) transcriptional factors were simultaneously up-regulated under non-endotoxemic condition. LPS injection significantly modulated the obligate anaerobe bacterial populations of the Bacteroidetes class, and altered the population sizes of the Clostridium perfringens and the Bacteroides fragilis subgroup. Hyperglycemia did not enhance these alterations of the microbiota evoked by LPS, although it did modify the bacterial populations of the L. reuteri subgroup and staphylococci in healthy condition without endotoxemia.

Conclusions: The present study indicates that both gut immune function and microbiota are significantly modulated by persistent hyperglycemia.

Keywords: Gut barrier function, Pro-inflammatory cytokine, CD4 ${ }^{+} \mathrm{T}$ lymphocyte subsets, Mesenteric lymph nodes

\section{Background}

Critically ill patients with persistent hyperglycemia are well known to show higher mortality rates, possibly due to the deterioration of a variety of cell functions $[1,2]$. Although the exact mechanisms remain to be fully clarified, functional alterations of the immune system and related cells could, at least in part, be responsible for the organ dysfunctions and poor outcomes of patients with hyperglycemia $[2,3]$. For example, hyperglycemia apparently alters the functions of immune cells such as monocytes, neutrophils and $\mathrm{T}$ or $\mathrm{B}$ lymphocytes $[2,4,5]$. In addition,

\footnotetext{
*Correspondence: takeshi-su@a7.keio.jp

'Department of Anesthesiology, Keio University School of Medicine, 35

Shinanomachi, Shinjuku-ku, Tokyo 160-8582, Japan

Full list of author information is available at the end of the article
}

hyperglycemia, by itself, is known to augment the production of inflammatory cytokines such as tumor necrosis factor- $\alpha$ (TNF- $\alpha$ ) and interlukin-6 (IL-6) [6].

Maintenance of the intestinal homeostasis and function is very important issue to improve the prognosis of critically ill patients, since intestinal barrier dysfunction has been shown to contribute to the deterioration of the host immune function. Among the gut immune systems, gut-associated lymphoid tissue (GALT) is of great consequence, not only in relation to regulation of the intestinal homeostasis, but also to the maintenance of systemic immunity [7]. In particular, mesenteric lymph nodes (MLNs) of GALT are considered as the key tissues for antigen-driven priming, activation and polarization of naive $\mathrm{T}$ cells towards several $\mathrm{T}$ cell subpopulations, 
such as helper T cell 1 (Th1), 2 (Th2), 17 (Th17) and regulatory $\mathrm{T}$ cells (Treg) $[7,8]$. As apparent overproduction of inflammatory cytokines has been found in the MLNs of critically ill conditions [9], it is likely that the normal MNL function could be complicated in critically ill patients. On the other hand, an enormous amount of bacteria, estimated at approximately $10^{12}$ commensal bacteria covering over 1,000 species, inhabit and together constitute independent bacterial ecosystems, known as microbiota, in the gut [10]. Although the microbiota contribute to the development of the host immune potential [7], its alterations in quality and quantity, called dysbiosis, have been shown to play substantial roles in gut inflammation and metabolic disease [11], and are associated with organ dysfunctions and poor outcomes in critically ill patients through causing gut barrier dysfunction [12].

We previously demonstrated that excessive hyperglycemia at levels of over $400 \mathrm{mg} / \mathrm{dL}$ for short-term periods, like $3 \mathrm{hr}$, did not evoke alterations of the gut microbiota, but apparently induced gut barrier dysfunction through TNF- $\alpha$-dependent pathways [13]. However, it remains to be determined if longer time period of hyperglycemia induce the change of the immune status of MLNs and the alterations of the microbiota, especially in the critically ill condition.

In the present study, we therefore hypothesized that persistent hyperglycemia for 24 hrs up-regulated the proinflammatory cytokines, and thereby promoted the differentiation of $\mathrm{CD} 4^{+}$lymphocytes to Th1 and Th17, which posses the ability of inducing inflammatory responses, in MLNs. Besides, we examined whether these alterations of the immune status in MLNs were followed by the disruption of the microbiota homeostasis; that is, increase of pathogens and decrease of probiotics. Furthermore, we proposed the $2 \times 2$ study design; control and hyperglycemia group with/ without eodotoxemia, to evaluate the effect of disease condition on the change induced by hyperglycemia. We expected that endotoxemia augmented the effect of hyperglycemia.

\section{Methods}

This study protocol was approved by the Animal Care and Committee of Keio University School of Medicine (KI002001) in accordance with the National Institute of Health guideline.

\section{Animal preparation}

Male Wistar rats (6-7 weeks), weighting 350-400 g, were fed standard chow and studied after an acclimation period of 3 to 7 days at our laboratory. Under isoflurane anesthesia in oxygen, the jugular vein and carotid artery were cannulated (PE50; Intermedic, Sparks, MD). Jugular vein was used for continuous infusion of glucose solution, whereas arterial line was used for continuous monitoring and blood sampling described below. After the preparatory surgery, the animals were placed in a metabolic cage that allowed for awakening and stabilization for $1 \mathrm{hr}$.

\section{Study protocol}

After the preparatory surgery and 1-hr stabilization period, 20 rats were randomly assigned to either the Control group (C: $n=10)$ or the Endotoxemia (E: $n=10)$ group, the animals in the latter of which were administered LPS $(1 \mathrm{mg} / \mathrm{kg})$ by intravenous injection. Based on our pilot study data, we chose this dose of LPS in which the mortality rate at $48 \mathrm{hrs}$ was approximately 10-15\% without hyperglycemia and 20-30\% with hyperglycemia. In other words, we intended to make our endotoxemia model not lethal but moderately damaged to mimic disease condition. Both groups were further divided into 2 subgroups ( $n=5$, each group): the Normoglycemia group (N: $80-150 \mathrm{mg} / \mathrm{dL}$ ), administered normal saline, and the Hyperglycemia group (H: $250-350 \mathrm{mg} / \mathrm{dL}$ ), administered glucose solution, resulting in four different groups ( $\mathrm{CN}, \mathrm{CH}, \mathrm{EN}$, and $\mathrm{EH}$ group). All solutions were infused at the rate of $10 \mathrm{ml} / \mathrm{kg} / \mathrm{hr}$ throughout the $24 \mathrm{hr}$ study period. In our pilot study, we confirmed that such infusion rate did not alter arterial $\mathrm{pH}$ and electrolytes significantly at least for $24 \mathrm{hrs}$. Blood sample $(10 \mu \mathrm{L})$ was obtained at $0,3,6,12$, and $24 \mathrm{hr}$ study period for measurement of blood glucose. In the Hyperglycemia group, all rats received $20 \%$ of glucose infusion at base line, and then, the concentration was adjusted according to the blood glucose levels measured at 3, 6, and $12 \mathrm{hr}$ time-points during the study period. If blood glucose level was more than 350 or $500 \mathrm{mg} / \mathrm{dL}$, the infusion concentration was reduced by $5 \%$ or $10 \%$ respectively. On the other hand, if blood glucose was less than 200 or $150 \mathrm{mg} / \mathrm{dL}$, the infusion concentration was increased by $5 \%$ or $10 \%$ respectively (maximum $40 \%$, minimum $10 \%$ of glucose infusion). Since one animal in the $\mathrm{EH}$ group died before the end of the study period, we added one rat in the $\mathrm{EH}$ group so that the number of animals in each group was equal $(n=5)$. At $24 \mathrm{hr}$, the MLNs were excised thorough laparotomy and the adipose tissue around it was removed for the measurement of inflammatory cytokine (tumor necrosis factor- $\alpha$ [TNF- $\alpha$ ], interleukin-1 $[$ [L-1 $\beta]$ and IL-6) mRNAs and levels of transcriptional factors of $\mathrm{CD}^{+} \mathrm{T}$ lymphocyte subsets (Th1, Th2, Th17 and Treg). Then, all tissues were incubated in RNAlater solution (Ambion Inc., Austin, TX, USA) overnight at $4{ }^{\circ} \mathrm{C}$ and stored at $-80{ }^{\circ} \mathrm{C}$ until the mRNA measurement by semi-quantitative real timepolymerase chain reaction (Semi-quantitative RT-PCR).

To clarify the alterations of the gut microbiota, another series of experiments were performed: Following the same methods described above, 26 rats were randomly divided into the same 4 groups $(\mathrm{CN}: n=6, \mathrm{CH}$ : $n=7, \mathrm{EN}: n=6, \mathrm{EH}: n=7)$ and received the same 
treatment during the next $24 \mathrm{hr}$ study period. One rat was added to the EH group, since one rat in this group died before the end of the study period. After laparotomy under anesthesia, the contents of colon were harvested from the whole colon, and sampled and weighed before suspension in RNAlater solution (Ambion Inc., Austin, TX, USA) and then stored at $4{ }^{\circ} \mathrm{C}$ until the analyses described below.

\section{Preparation of total RNA for evaluation of the cytokines and $\mathrm{CD}^{+} \mathrm{T}$ lymphocyte subsets}

We performed semi-quantitative RT-PCR to examine the mRNA level of each of the inflammatory cytokines and $\mathrm{CD}_{4}^{+} \mathrm{T}$ lymphocyte master genes in the MLNs. The total RNA from the MLNs was extracted using RiboPur$\mathrm{e}^{\mathrm{TM}}$ kit (Ambion, CA, USA). Briefly, $50 \mathrm{mg}$ of minced MLNs was transferred to $1.5-\mathrm{ml}$ sterile tubes containing microbeads. Then, 1-ml TRI regent (Ambion, CA, USA), a monophasic solution containing phenol and guanidine thiocyanate, was added to the tubes, followed by homogenization using a tissue homogenizer (Micro Smash-100R, Tomy, Japan) at 2,000 x g for $2 \mathrm{~min}$ at $4^{\circ}$ C. After the homogenization, each sample was centrifuged at $4,500 \mathrm{x}$ g for $10 \mathrm{~min}$ at $4{ }^{\circ} \mathrm{C}$ and the supernatants were transferred to $1.5-\mathrm{ml}$ sterile tubes. Then, $100 \mu \mathrm{L}$ of 1 -Bromo-3-Chloropropane, a phase separation reagent, was added to the supernatants, followed by incubation and centrifugation at 4,500 x g for $10 \mathrm{~min}$ at $4{ }^{\circ} \mathrm{C}$, and transfer of the aqueous phase to 1.5 - $\mathrm{mL}$ sterile tubes, to which $200 \mu \mathrm{L}$ of $100 \%$ ethanol was added. Thereafter, the samples were transferred into $1.5-\mathrm{mL}$ sterile tubes with a filter cartridge, and centrifuged at $8,000 \mathrm{rpm}$ for $10 \mathrm{~min}$ at $4{ }^{\circ} \mathrm{C}$. The filter cartridges containing total RNA were washed twice with wash solution, followed by addition of $100 \mu \mathrm{L}$ of elution buffer and centrifugation for $30 \mathrm{sec}$. The quantity and quality of the eluted total RNA were measured using the NanoDrop 1000A spectrophotometer (Thermo Fisher Scientific-NanoDrop products, DE, USA), followed by dilution of the RNA samples with nuclease-free water (Ambion, CA, USA). To remove contaminating DNA from the isolated RNA, DNase treatment was performed using a DNA-free kit (Ambion, CA, USA). The complementary DNA (cDNA) synthesis from the total RNA was performed using the ReverTra Ace qPCR RT kit (Toyobo, Tokyo, Japan) with random primers, in accordance with the instructions supplied by the manufacturer.

\section{Determination of the mRNA expression levels of cytokines and $\mathrm{CD}^{+} \mathrm{T}$ lymphocyte master genes by semi- quantitative-RT-PCR}

The target gene expressions were analyzed by semiquantitative RT-PCR using TaqMan-based Applied Biosystem gene expression assays (Applied Biosystem, CA,
USA). The primers and probes designed for this system were as follows; Rn9999017_m1 (Tnf) for TNF- $\alpha$, Rn00580432_m1 (Il1b) for IL-1ß, Rn01410330_m1 (Il6) for IL-6, Rn01461633_m1 (Tbx21) for Th1 transcriptional factors, Rn00484683_m1 (Gata3) for Th2 transcriptional factors, Rn01533717_g1 (Rorc) for Th17 transcriptional factors, Rn01525092_m1 (Foxp3) for Treg transcriptional factors, and Rn00607869_m1 (Actb) for $\beta$-actin. Quantification was normalized to the endogenous expression of the $\beta$-actin gene. The reaction mixture and PCR cycles were adjusted according to the manufacturer's instructions (Applied Biosystem, CA, USA). The data were analyzed using the OneStep Software (Applied Biosystem, CA, USA). After determining the comparative threshold cycle $\left(C_{T}\right)$ values for $\beta$-actin as reference, the relative quantifications were carried out using the $2^{-\Delta \Delta C t}$ method and standardized by the $C_{T}$ value for $\beta$-actin. The data were expressed as mean \pm standard deviation.

\section{Isolation of total RNA for the microbiota analysis and bacterial count by quantitative RT-PCR}

After the intestinal contents of the colon were homogenized using a mortar on ice, an aliquot of fecal homogenate $(20 \mu \mathrm{L})$ was added to $1 \mathrm{~mL}$ sterilized PBS solution and centrifuged at 5,000 x g for $10 \mathrm{~min}$. The supernatant was discarded and RNA was isolated using a modification of the guanidinium thiocyanate-phenolchloroform extraction method $[14,15]$. To quantify the bacteria present in the samples, we examined the gut microbiota composition by the $16 \mathrm{~S}$ rRNA-targeted quantitative RT-PCR kit, Yakult Intestinal Flora-SCAN $\left(\right.$ YIF-SCAN $\left.^{\circ}\right)$ [14, 15]. Quantitative RT-PCR was conducted in a one-step reaction using a Qiagen OneStep RT-PCR kit (Qiagen GmbH, Hilden, Germany) and SYBR green method (Molecular Probes, Eugene, OR). The products of reaction mixture, reverse transcription and PCR cycles were assessed as previously described $[14,15]$. A standard curve was generated using the $C_{T}$ values of quantitative RT-PCR data and the corresponding cell counts, determined microscopically with 4,6-diamidino-2-phe-nylindole (DAPI) (Vector Laboratories, Burlingame, CA) staining methods, of the dilution series of the standard strains. In the present study, we measured the various types of bacteria in the gut microbiota by using specific primer sets, including major types of bacteria in the intestine, such as Enterococcus, and Enterobacteriaceae to which E.coli belong. To determine the number of bacteria present in the samples, 3 serial 10 -fold dilutions of the extracted RNA sample (corresponding to $1 / 2,000,1 / 20,000$, and $1 / 200,000$ of the amount of RNA extracted from $20 \mathrm{mg}$ of colon contents) were used for the quantitative RT-PCR, and the $C_{T}$ values in the linear range of the assay were applied to 
the standard curve generated in the same experiment to obtain the corresponding bacterial cell counts in each nucleic acid sample. The specificity of the quantitativeRT-PCR assay using group- or species-specific primers was determined as described previously $[14,15]$

\section{Statistical analyses}

All data were expressed as mean $\pm \mathrm{SD}$ after confirmation of normal distribution. The repeated-measures data of blood glucose obtained at various time-points were analyzed by mixed-effects analysis of variance (ANOVA) using the statistical software package, EXCEL-Longitudinal Data Analysis, Ver1.0 for Windows (ESUMI, Tokyo, Japan). Then, Post-hoc test was performed using Tukey's test. Other data, such as the mRNA levels detected by RT-PCR were analyzed by two-way ANOVA for interaction between two main effects of endotoxemia (Factor A; C vs E) and hyperglycemia (Factor B; N vs $\mathrm{H}$ ). Ninety five percent of confidence interval for each four factors $(\mathrm{C}, \mathrm{E}, \mathrm{N}$, and $\mathrm{H})$ was also presented. When the interaction between two factors didn't exist, independent effects of these two factors were presented. If the interaction were detected, comparison of two groups were performed using Student's t-test separately in the Control group $(\mathrm{CN}$ vs $\mathrm{CH})$ and the Endotoxemia group (EN vs $\mathrm{EH}$ ) to elucidate the effect of hyperglycemia in both healthy and endotoxemic condition. The statistical analyses for mRNA were performed using SPSS/17.0 J (SPSS Inc, Chicago, IL). If mRNA was not detectable by RT-PCR, the value was regarded as zero. Differences at two-sided p-values of less than 0.05 were considered to be statistically significant.

\section{Results}

Changes of the blood glucose concentrations during the study period

Figure 1 indicates the changes of the blood glucose levels over the $24 \mathrm{hr}$ study period. While the blood glucose levels in both the normoglycemic (CN and EN) groups were approximately in the range of $80 \mathrm{mg} / \mathrm{dL}$ to $120 \mathrm{mg} / \mathrm{dL}$, the hyperglycemic groups $(\mathrm{CH}$ and $\mathrm{EH})$ showed persistent hyperglycemia of approximately 250 to $350 \mathrm{mg} / \mathrm{dL}$ throughout the $24 \mathrm{hr}$ study period.

\section{Alterations of the mRNA expressions of the inflammatory cytokines and $\mathrm{CD}^{+} \mathrm{T}$ lymphocyte subsets in the MLNs}

No interaction between hyperglycemia and endotoxemia was found in the context of the expression of TNF- $\alpha$, IL- $1 \beta$ or IL- 6 mRNA in the MLNs. Furthermore, significant differences were observed between the hyperglycemic and normoglycemic groups in the expression levels of all of these cytokines. For instance, hyperglycemia per se, regardless of the presence/absence of concurrent endotoxemic insult, depressed the expression of TNF- $\alpha$ mRNA, but augmented the IL- $1 \beta$ and IL-6 mRNA expression in MLNs (Fig. 2a, b and c).

In the analysis of the transcriptional factors of $\mathrm{CD} 4^{+} \mathrm{T}$ lymphocytes, the interaction between endotoxemia and hyperglycemia was not statistically significant in the effect on Tbx21 (Fig. 3a) or Rorc (Fig. 3c), but was in the effect on Gata3 (Fig. 3b) and Foxp3 (Fig. 3d). There were

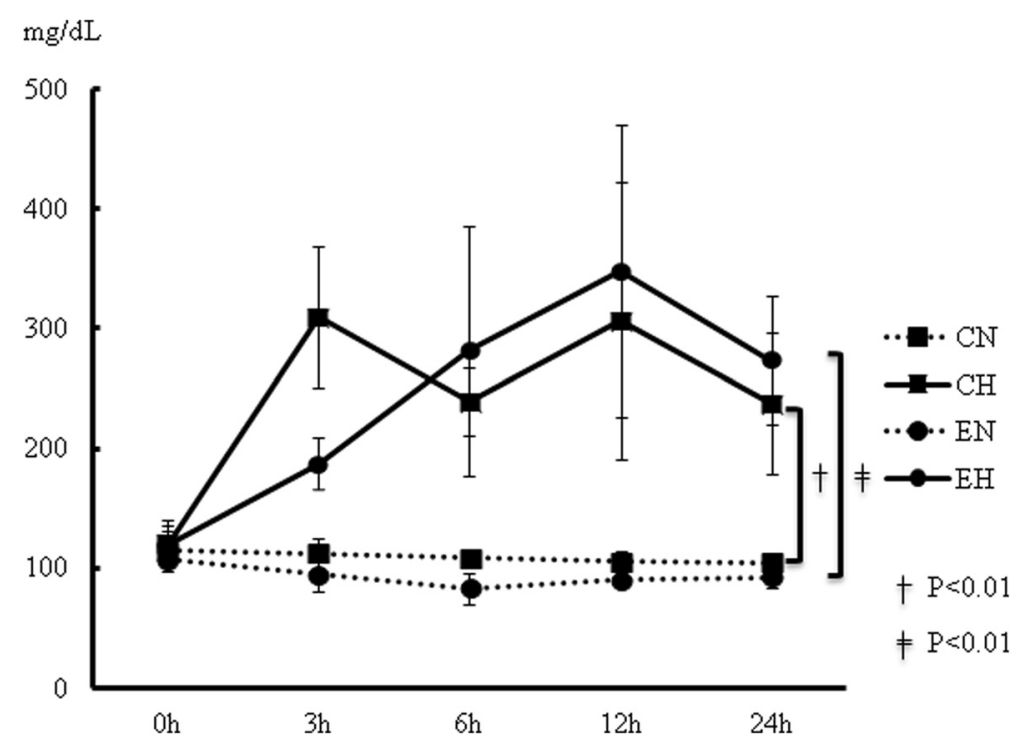

Fig. 1 Changes of blood glucose concentrations during 24 hr study period. Comparison between four groups was performed by mixed-effects analysis of variance (ANOVA) followed by Tukey's post hoc test. Abbreviation; CN: control with normoglycemia group, CH: control with hyperglycemia group, EN: endotoxemia with normoglycemia group, EH: endotoxemia with hyperglycemia group. $\dagger P<0.01: \mathrm{CH}$ versus $\mathrm{CN}, \neq$ : $P<0.01 \mathrm{EH}$ versus EN. Data are expressed as mean \pm SD 

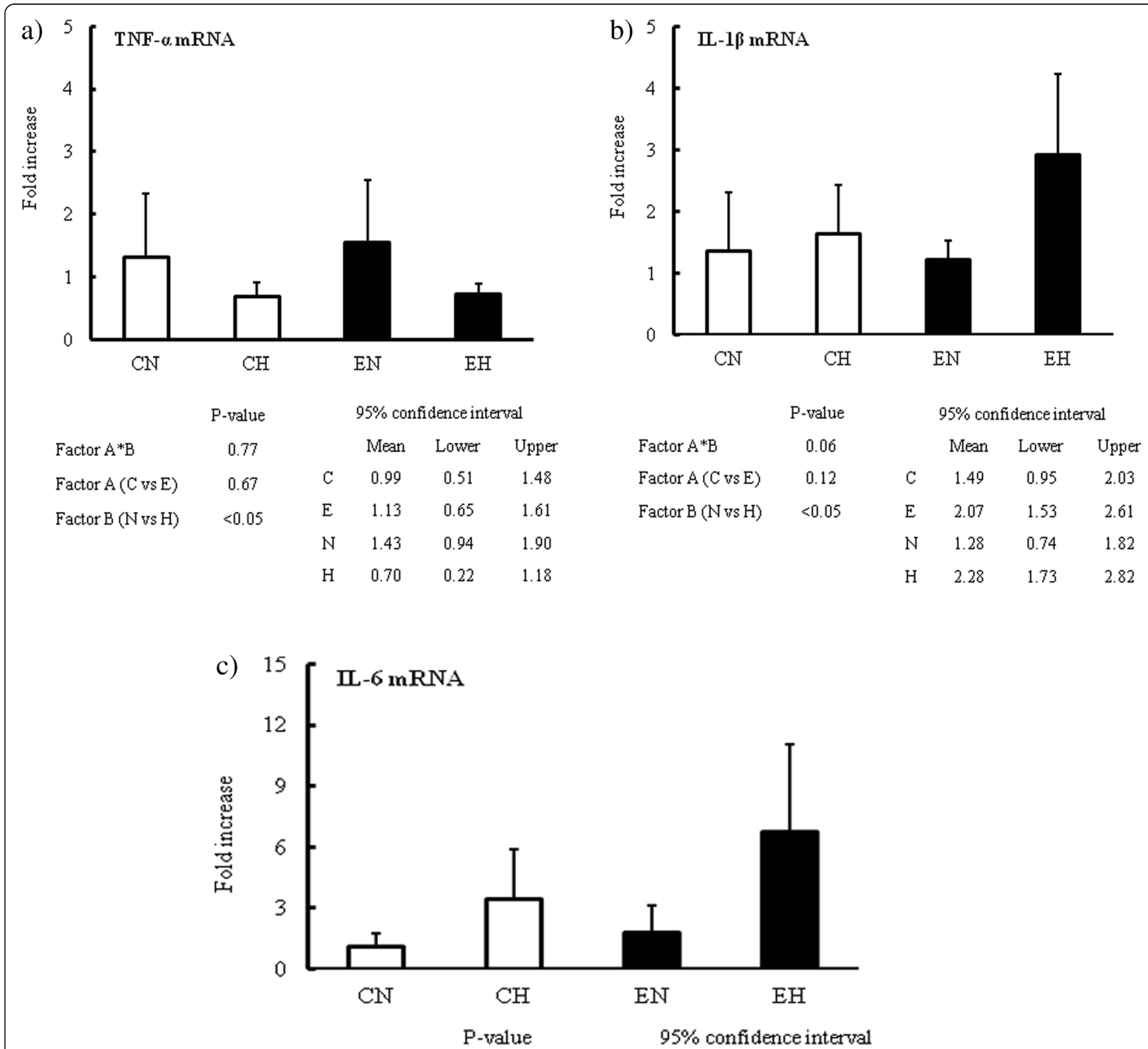

$\begin{array}{lccccc}\text { Factor } \mathrm{A}^{*} \mathrm{~B} & 0.20 & & \text { Mean } & \text { Lower } & \text { Upper } \\ \text { Factor A (C vs E) } & 0.06 & \mathrm{C} & 2.27 & 0.86 & 3.69 \\ \text { Factor B (N vs H) } & <0.01 & \text { E } & 4.27 & 2.86 & 5.69 \\ & & \text { N } & 1.45 & 0.3 & 2.86 \\ & & \text { H } & 5.1 & 3.69 & 6.52\end{array}$

Fig. 2 Changes of TNF $-a, I L-1 \beta$ and IL-6 mRNA expression in MLNs. a) Expression of TNF-a mRNA at 24 hr study periods in MLNs. b) Expression of IL-1 $\beta$ mRNA at 24 hr study periods in MLNs. c) Expression of IL-6 mRNA at 24 hr study periods in MLNs. Inflammatory cytokines were analyzed by two-way ANOVA for interaction between factor A (control versus endotoxemia) and factor B (normoglycemia versus hyperglycemia). Ninety five percent confidence intervals for each four factors were also presented. If the interaction between two factors didn't exist, independent effects of these two factors were confirmed. If the interaction existed, comparison of two groups were performed using Student's t-test separately in the Control group (CN vs $\mathrm{CH}$ ) and the Endotoxemia group (EN vs EH). Abbreviation; CN: control with normoglycemia group, $\mathrm{CH}$ : control with hyperglycemia group, EN: endotoxemia with normoglycemia group, EH: endotoxemia with hyperglycemia group. Data are expressed as mean \pm SD. White and black bars indicate control and endotoxemia group, respectively 

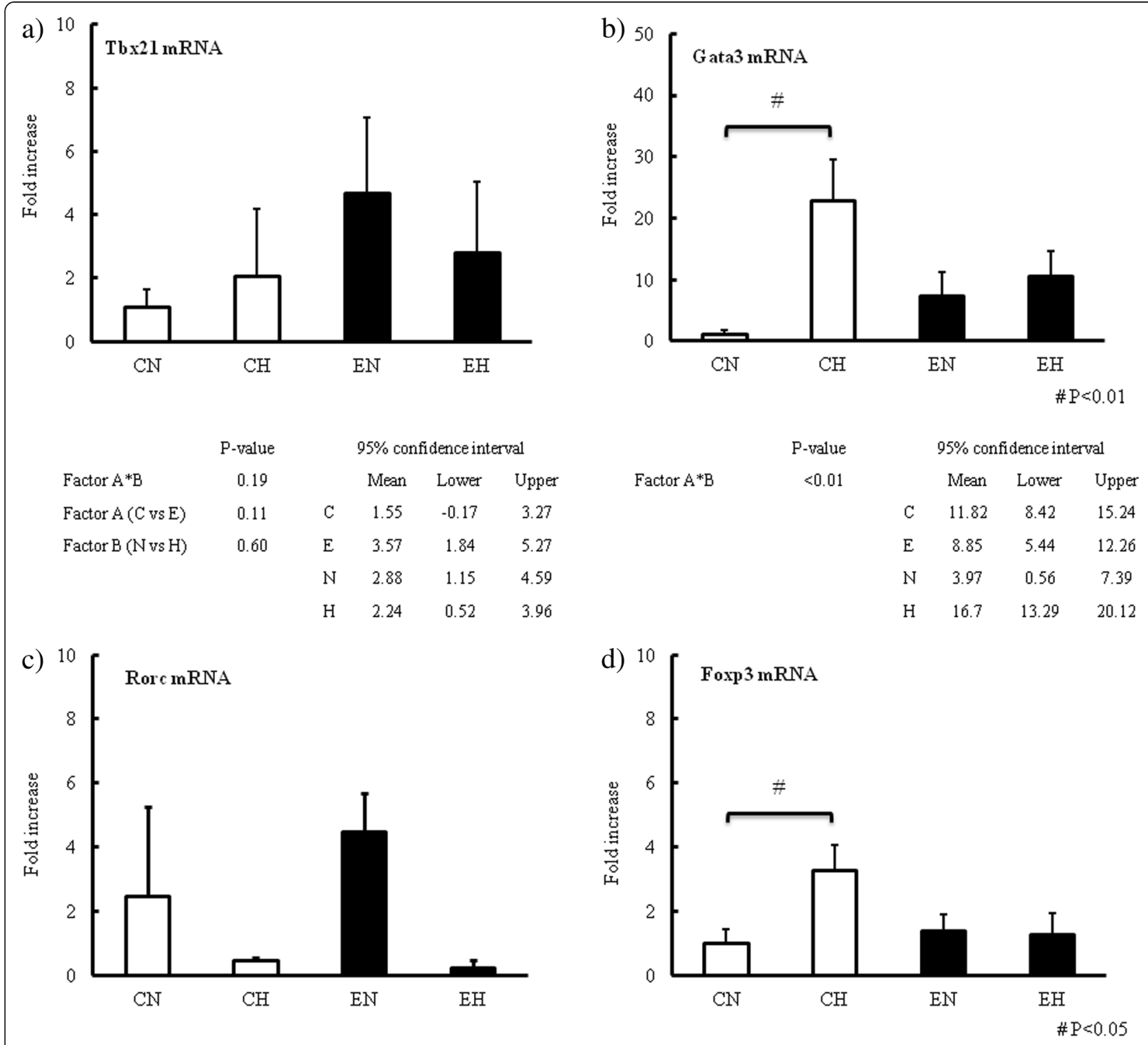

\begin{tabular}{|c|c|c|c|c|c|c|c|c|c|c|c|}
\hline \multirow[b]{2}{*}{ Factor $A^{*} B$} & \multirow{2}{*}{$\begin{array}{c}\text { P-value } \\
0.71\end{array}$} & & \multicolumn{3}{|c|}{$95 \%$ confidence interval } & \multirow[b]{2}{*}{ Factor $\mathrm{A}^{*} \mathrm{~B}$} & \multirow{2}{*}{$\begin{array}{l}\text { P-value } \\
<0.01\end{array}$} & \multicolumn{4}{|c|}{$95 \%$ confidence interval } \\
\hline & & & Mean & Lower & Upper & & & & Mean & Lower & Upper \\
\hline Factor $A(C$ vs $E)$ & 0.65 & $\mathrm{C}$ & 1.39 & -0.04 & 2.82 & Factor $A(C$ vs $E)$ & $<0.05$ & $\mathrm{C}$ & 2.14 & 1.55 & 2.71 \\
\hline \multirow{3}{*}{ Factor $\mathrm{B}(\mathrm{N}$ vs $\mathrm{H})$} & 0.15 & $E$ & 0.92 & -052 & 2.35 & Factor $\mathrm{B}(\mathrm{N}$ vs $\mathrm{H})$ & $<0.05$ & $\mathrm{E}$ & 1.32 & 0.74 & 1.91 \\
\hline & & $\mathrm{N}$ & 1.90 & 0.46 & 3.33 & & & $\mathrm{~N}$ & 1.19 & 0.61 & 1.78 \\
\hline & & $\mathrm{H}$ & 0.41 & -1.02 & 1.84 & & & $\mathrm{H}$ & 2.26 & 1.68 & 2.84 \\
\hline
\end{tabular}

Fig. 3 Changes of Tbx21, Gata3, Rorc and Foxp3 mRNA expression in MLNs. a) Expression of Tbx21 mRNA at 24 hr study period in MLNs. b) Expression of Gata3 mRNA at 24 hr study period in MLNs. c) Expression of Rorc mRNA at 24 hr study period in MLNs. d) Expression of Foxp3 mRNA at $24 \mathrm{hr}$ study period in MLNs. Four types of transcriptional factors for CD4+ T lymphocytes were analyzed by two-way ANOVA for interaction between factor A (control versus endotoxemia) and factor B (normoglycemia versus hyperglycemia). Ninety five percent confidence intervals for each four factors were also presented. If the interaction between two factors didn't exist, independent effects of these two factors were confirmed. If the interaction existed, comparison of two groups were performed using Student's t-test separately in the Control group (CN vs CH) and the Endotoxemia group (EN vs EH). Abbreviation; CN: control with normoglycemia group, $\mathrm{CH}$ : control with hyperglycemia group, EN: endotoxemia with normoglycemia group, EH: endotoxemia with hyperglycemia group. Data are expressed as mean \pm SD. White and black bars indicate control and endotoxemia group, respectively. P values described in the figure indicates those obtained by post hoc test 
no independent significant effects of endotoxemia or hyperglycemia on Tbx21 and Rorc mRNA. Regarding Gata3 and Foxp3 mRNA, hyperglycemia induced a significant augmentation of Gata3 (Fig. 3c, $p<0.01$ ) and Fox3 mRNA (Fig. 3d, $p<0.05$ ) in the Control group only, suggesting that hyperglycemia could induce the transcription of Gata3 and Foxp3 as representatives of Th2 and Treg in the MLNs in the healthy condition, not the endotoxemic condition.

\section{Alterations of the intestinal microbiota}

To clarify whether persistent hyperglycemia evoked by exogenous glucose infusion for 24 hrs modulates the intestinal microbiota differently in the presence or absence of endotoxemia, we evaluated the changes in the populations of the obiligate/facultative anaerobes and aerobes by quantitative RT-PCR. The classification of bacteria we evaluated in this study is shown in the Additional file 1: Figure S1.

There were no significant differences in the total populations of obligate/facultative anaerobes and aerobes in the colon contents among the groups (Fig. 4). In the class level analysis, however, the independent main effect of endotoxemia was found on the Bacteroidetes class (decrease), even though hyperglycemia had no independent effects (Fig. 4). Table 1 shows the alteration of each type of bacteria in the colon microbiota, presented as the logarism 10 cells/g of colon contents. In the lower-level analyses than the class level, endotoxemia induced the increase of Clostridium perfringens and the decrease of Bacteroides fragilis subgroups independently regardless of the existence of hyperglycemia, while hyperglycemia itself decreased the Lactobacillus ruminis subgroup without the interaction effect of endotoxemia. The interaction between the two factors (hyperglycemia and endotoxemia) was found on the Lactobacillus reuteri subgroup, staphylococci, enterococci and Enterobacteriaceae (Table 1). In the Control group (healthy condition), hyperglycemia induced the decrease of Lactobacillus reuteri subgroup, and the increase of staphylococci. On the other hand, hyperglycemia decreased both enterococci and Enterobacteriaceae in the Endotoxemia group (endotoxemic condition).

Collectively, hyperglycemia and endotoxemia didn't alter the gut microbiota synergically, even though both hyperglycemia and endotoxemia independently modified the proportions of the obligate and facultative anaerobes in the gut microbiota. In some type of bacteria, the effect of hyperglycemia differed between healthy and endotoxemic condition.

\section{Discussion}

The present study demonstrated that persistent hyperglycemia induced by exogenous glucose infusion altered the gut immune functions and microbiota in the colon. Another important finding was that hyperglycemia per se could independently alter the mRNA expression levels of inflammatory cytokines in the MLNs. In particular, the mRNA expressions of IL- $1 \beta$ and IL- 6 were augmented regardless of endotoxemia condition, and those of $\mathrm{CD}_{4}^{+} \mathrm{T}$ lymphocyte master genes, such as Gata3 and Foxp3, were increased under healthy condition without endotoxemia in the MLNs, following exposure to prolonged state of hyperglycemic insult for 24 hours. While endotoxemia is known to modulate the distribution of some obligate and facultative anaerobes, including both pathogenic and non-pathogenic bacteria, the present study indicates that hyperglycemia also evoked alterations of the gut microbiota, such as changes in the populations of the Lactobacillus reuteri subgroup and staphylococci in the colon under healthy condition.

MLNs, the GALT tissue of greatest consequence, play a key role in the gut and host immune systems [7], and simultaneously regulate both local and systemic inflammatory responses [9]. In the present study, we evaluated the expression of pro-inflammatory cytokines such as TNF- $\alpha$, IL- $1 \beta$, and IL-6, representative markers of the early phase of critically ill. The present finding that persistent hyperglycemia for 24 hrs independently upregulated the mRNA expressions of such inflammatory cytokines except for TNF- $\alpha$ in the MLNs indicates that hyperglycemic state induced by exogenous glucose infusion per se can activate the MLNs to modulate the host immune responses. The discharges of TNF- $\alpha$ secretion might have been peaked-out at this moment in this experimental model. While several mechanisms underlying the activation of MLNs by continuous hyperglycemic state could be proposed, macrophages residing in the MLNs [16] are considered as the major source of production of inflammatory cytokines [17]. In a previous study, hyperglycemia over the level of $315 \mathrm{mg} / \mathrm{dL}$ could directly impair the functions of activated macrophages, resulting in up-regulation of the mRNA expressions of IL-1 $\beta$ and IL-6 [18]. Collectively, hyperglycemia persisting for a day can augment the inflammatory responses in the MLNs via activation of macrophages, thereby modulating the functions of the immune system and organ functions.

$\mathrm{CD} 4^{+}$lymphocytes also contribute to the preservation of immune homeostasis and inflammatory responses in the gut [7]. We examined the differentiation of $\mathrm{CD} 4^{+}$ lymphocytes by measuring the master genes, including the Tbx21 for Th1, the Rorc for Th17, Gata3 for Th2, and Foxp3 for Treg to evaluate whether hyperglycemia and endotoxemia synergistically modulate the balance between pro- and anti-inflammatory responses. Previous study showed that both Th1 and Th17 cells were responsible for the release of inflammatory cytokines [19], 
a)
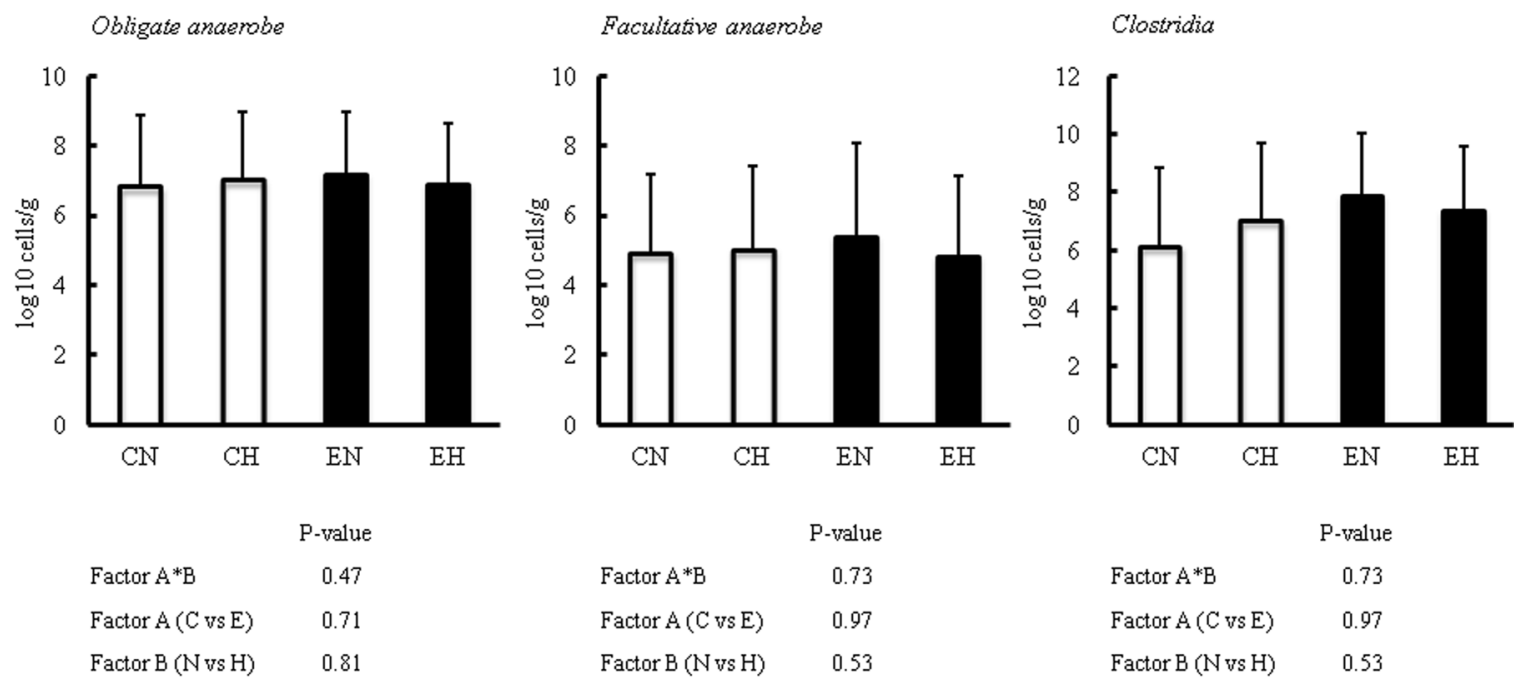

\begin{tabular}{cccc}
\multicolumn{4}{c}{$95 \%$ confidence interval } \\
& Mean & Lower & Upper \\
C & 6.91 & 6.49 & 7.34 \\
E & 7.03 & 6.60 & 7.45 \\
N & 7.01 & 6.58 & 7.43 \\
H & 6.94 & 6.51 & 7.36
\end{tabular}

\begin{tabular}{|c|c|c|c|}
\hline \multicolumn{3}{|c|}{ Factor $A * B$} & 0.73 \\
\hline \multicolumn{3}{|c|}{ Factor $A(C$ vs $E)$} & 0.97 \\
\hline \multicolumn{3}{|c|}{ Factor $\mathrm{B}(\mathrm{N}$ vs $\mathrm{H})$} & 0.53 \\
\hline \multicolumn{4}{|c|}{$95 \%$ confidence interval } \\
\hline & Mean & Lower & Upper \\
\hline $\mathrm{C}$ & 4.49 & 3.99 & 4.98 \\
\hline $\mathrm{E}$ & 4.47 & 3.98 & 4.97 \\
\hline$N$ & 4.59 & 4.09 & 5.09 \\
\hline $\mathrm{H}$ & 4.37 & 3.88 & 4.86 \\
\hline
\end{tabular}

b)
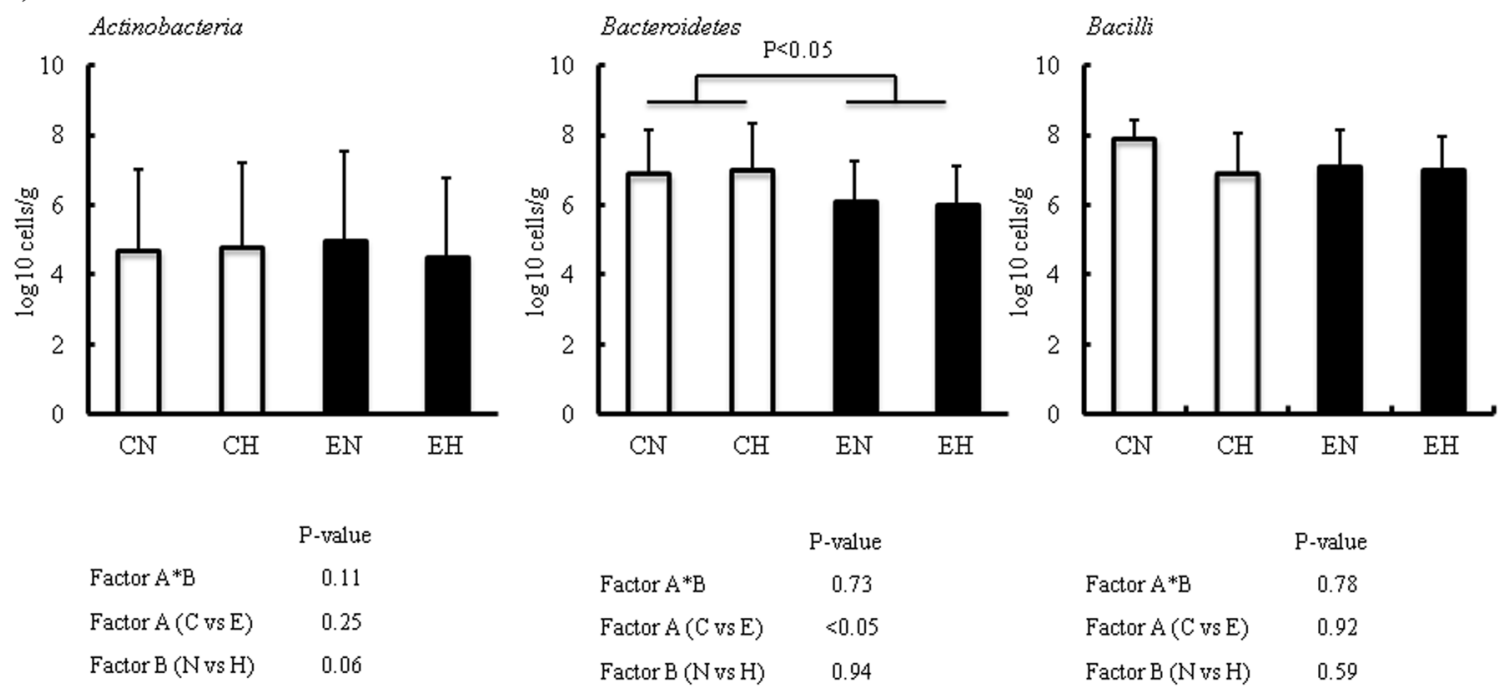

\begin{tabular}{cccc} 
& \multicolumn{3}{c}{$95 \%$ confidence interval } \\
& Mean & Lower & Upper \\
C & 7.40 & 7.00 & 7.81 \\
E & 7.08 & 6.67 & 7.49 \\
N & 7.50 & 7.09 & 7.91 \\
H & 6.98 & 6.57 & 7.39
\end{tabular}

\begin{tabular}{cccc} 
& \multicolumn{3}{c}{$95 \%$ confidence interval } \\
& Mean & Lower & Upper \\
C & 6.96 & 6.39 & 7.52 \\
E & 6.04 & 5.47 & 6.60 \\
N & 6.51 & 5.95 & 7.07 \\
H & 6.48 & 5.92 & 7.04
\end{tabular}

Fig. 4 (See legend on next page.) 
(See figure on previous page.)

Fig. 4 Alterations of obligate and facultative anaerobes, individual bacteria classes in colon contents. Alterations of obligate and facultative anaerobes, individual bacteria classes in colon contents were analyzed by two-way ANOVA for interaction between factor A (control versus endotoxemia) and factor B (normoglycemia versus hyperglycemia). Ninety five percent confidence intervals for each four factors were also presented. If the interaction between two factors didn't exist, independent effects of these two factors were confirmed. If the interaction existed, comparison of two groups were performed using Student's t-test separately in the Control group (CN vs CH) and the Endotoxemia group (EN vs EH). The main effect of endotoxemic insult was found only in class Bcteroidetes. Abbreviation; CN: control with normoglycemia group, CH: control with hyperglycemia group, EN: endotoxemia with normoglycemia group, EH: endotoxemia with hyperglycemia group. Data are expressed as mean \pm SD (log10 cells/g of colon contents). White and black bars indicate control and endotoxemia group, respectively

whereas Th2 and Treg for the production of antiinflammatory cytokines such as IL-4 and IL-10 [19, 20]. Contrary to our initial hypothesis, the present study showed that persistent hyperglycemia evoked by exogenous glucose infusion evoked up-regulation of the Th2(Gata3) and Treg (Foxp3) master genes in favor of Th1 (Tbx21) and Th17 (Rorc) in healthy condition, which was not found in the endotoxemic condition. Recent evidence has indicated that $\mathrm{T}$ cell function and differentiation, including increased Th1 (inflammatory) to Th2 (anti-inflammatory) differentiation and increased Treg cells were associated with the development of immunosuppression in the late phase of sepsis [21, 22]. Thus, hyperglycemia-induced up-regulation of Th2 and Treg found in our study could, in turn, lead to immunosuppressive status, possibly resulting in the poor outcome in

Table 1 Alteration of lower than class level bacteria in colon microbiota

\begin{tabular}{|c|c|c|c|c|c|c|c|}
\hline & $\begin{array}{l}\text { CN group } \\
\text { (detection rate) }\end{array}$ & $\begin{array}{l}\text { CH group } \\
\text { (detection rate) }\end{array}$ & $\begin{array}{l}\text { EN group } \\
\text { (detection rate) }\end{array}$ & $\begin{array}{l}\text { EH group } \\
\text { (detection rate) }\end{array}$ & $\begin{array}{l}\text { Factor A (C } \\
\text { vs E) }\end{array}$ & $\begin{array}{l}\text { Factor B (N } \\
\text { vs H) }\end{array}$ & $\begin{array}{l}\text { Factor } \\
A^{*} B\end{array}$ \\
\hline Total bacterial counts & $9.4 \pm 0.2(6 / 6)$ & $9.8 \pm 0.8(7 / 7)$ & $10.1 \pm 0.4(6 / 6)$ & $9.7 \pm 1.1(7 / 8)$ & $P=0.43$ & $P=0.98$ & $P=0.31$ \\
\hline \multicolumn{8}{|l|}{ Obligate anaerobe } \\
\hline $\begin{array}{l}\text { Clostridium coccoides } \\
\text { group }\end{array}$ & $7.8 \pm 0.3(6 / 6)$ & $8.6 \pm 0.5(7 / 7)$ & $9.1 \pm 0.4(6 / 6)$ & $8.7 \pm 1.1(7 / 7)$ & $P=0.10$ & $P=0.67$ & $P=0.11$ \\
\hline C. leptum subgroup & $8.2 \pm 0.5(6 / 6)$ & $8.9 \pm 0.7(7 / 7)$ & $9.5 \pm 0.3(6 / 6)$ & $8.8 \pm 1.1(7 / 7)$ & $P=0.17$ & $P=0.99$ & $P=0.12$ \\
\hline C. perfringens & $<2.3(6 / 6)$ & $3.5 \pm 1.6(4 / 7)$ & $5.1 \pm 1.2(6 / 6)$ & $4.7 \pm 1.1(6 / 7)$ & $P<0.01$ & $P=0.39$ & $P=0.12$ \\
\hline Bifidobacterium & $7.7 \pm 0.6(6 / 6)$ & $6.4 \pm 1.2(7 / 7)$ & $6.4 \pm 1(5 / 6)$ & $6.5 \pm 0.9(6 / 7)$ & $P=0.09$ & $P=0.13$ & $P=0.06$ \\
\hline Atopobium cluster & $8.1 \pm 0.4(6 / 6)$ & $7.4 \pm 1(6 / 7)$ & $7.7 \pm 0.5(6 / 6)$ & $7.6 \pm 0.8(7 / 7)$ & $P=0.1$ & $P=0.16$ & $P=0.57$ \\
\hline Prevotella & $5.9 \pm 1.1(3 / 6)$ & $6.4 \pm 1.4(4 / 7)$ & $7.2 \pm 0.4(6 / 6)$ & $6.6 \pm 1.1(7 / 7)$ & $P=0.12$ & $P=0.90$ & $P=0.24$ \\
\hline $\begin{array}{l}\text { Bacteroides fragilis } \\
\text { group }\end{array}$ & $7.9 \pm 0.5(6 / 6)$ & $7.6 \pm 1.2(7 / 7)$ & $<5.0(0 / 6)$ & $7.2(1 / 7)$ & $P<0.01$ & $P=0.97$ & $P=0.44$ \\
\hline \multicolumn{8}{|l|}{ Facultative anaerobe } \\
\hline L. gasseri subgroup & $9.1 \pm 0.5(6 / 6)$ & $9.5 \pm 1.2(7 / 7)$ & $9.9 \pm 0.6(6 / 6)$ & $9.4 \pm 1.3(7 / 7)$ & $P=0.46$ & $P=0.97$ & $P=0.41$ \\
\hline L. brevis & $<2.3(6 / 6)$ & $2.9 \pm 1(2 / 7)$ & $3.4 \pm 0.8(6 / 6)$ & $3.0 \pm 0.9(3 / 7)$ & $P=0.08$ & $P=0.75$ & $P=0.19$ \\
\hline L. casei subgroup & $<3.2(0 / 6)$ & $<3.2(0 / 7)$ & $<3.2(0 / 6)$ & $<3.2(0 / 7)$ & - & - & - \\
\hline L. fermentum & $<4.0(0 / 6)$ & $<4.0(0 / 7)$ & $<4.0(0 / 6)$ & $<4.0(0 / 7)$ & - & - & - \\
\hline $\begin{array}{l}\text { L. plantarum } \\
\text { subgroup }\end{array}$ & $<2.9(0 / 6)$ & $<2.9(0 / 7)$ & $<2.9(0 / 6)$ & $<2.9(0 / 7)$ & - & - & - \\
\hline L. reuteri subgroup & $7.7 \pm 0.7(6 / 6)$ & $4.9 \pm 2.8(4 / 7) *$ & $2.8 \pm 0.9(4 / 6)$ & $2.8 \pm 0.8(5 / 7)$ & - & - & $P<0.05$ \\
\hline L. ruminis subgroup & $4.5 \pm 0.8(6 / 6)$ & $4.4 \pm 1.1(6 / 7)$ & $7.2 \pm 1.3(6 / 6)$ & $5.3 \pm 1.7(7 / 7)$ & $P=0.07$ & $P<0.01$ & $P=0.05$ \\
\hline L. sakei subgroup & $2.5 \pm 0.4(3 / 6)$ & $2.8 \pm 0.6(6 / 7)$ & $2.9 \pm 0.7(5 / 6)$ & $2.9 \pm 0.8(5 / 7)$ & $P=0.38$ & $P=0.69$ & $P=0.57$ \\
\hline Staphylococcus & $5.1 \pm 0.7(6 / 6)$ & $6.7 \pm 1(7 / 7)^{* *}$ & $6.3 \pm 0.7(6 / 6)$ & $5.4 \pm 1.4(6 / 7)$ & - & - & $P<0.01$ \\
\hline Enterococcus & $6.7 \pm 1(6 / 6)$ & $7 \pm 0.7(7 / 7)$ & $7.8 \pm 0.7(6 / 6)$ & $6.8 \pm 0.8(7 / 7) * * *$ & - & - & $P<0.05$ \\
\hline Enterobacteriaceae & $6.6 \pm 1(6 / 6)$ & $7 \pm 1.5(6 / 7)$ & $8.8 \pm 0.3(6 / 6)$ & $7.6 \pm 0.6(7 / 7)^{* * * *}$ & - & - & $P<0.05$ \\
\hline \multicolumn{8}{|l|}{ Aerobes } \\
\hline Pseudomonas species & $<3.0(0 / 6)$ & $<3.0(0 / 7)$ & $<3.0(0 / 6)$ & $<3.0(0 / 7)$ & - & - & - \\
\hline
\end{tabular}

The numbers are presented as logalithm ${ }_{10}$ cells $/ g$ of colon contents

Data indicates mean \pm SD ( $\log 10$ cells $/ g$ of colon contents)

${ }^{*}: P<0.05$ versus CN group, ${ }^{* *}: P<0.01$ versus $C N$ group, ${ }^{* * *}: P<0.05$ versus EN group, ${ }^{* * * *}: P<0.01$ versus EN group 
critically ill. Although exact mechanisms to account for hyperglycemia-augmented $\mathrm{CD}_{4}^{+}$lymphocyte differentiation to Th2 and Treg was not clarified in our study, endogenous insulin could affect the differentiation of activated $\mathrm{CD}^{+}$lymphocytes, leading to the promotion of Th1 to Th2 differentiation [23], and simultaneously enhance the expression of Foxp3, well recognized as a Treg cell marker [24]. Since our model could be hyperinsulinemic due to prolonged state of hyperglycemia, as previously reported $[13,25]$, the alterations of Gata3 (Th2) and Foxp3 (Treg) expression found in the present study could be indirectly induced through an insulindependent pathway.

Disruption of the bacterial community in the gut may be associated with overgrowth of and colonization by pathogenic bacteria, which usually inhabit the intestinal tract in small numbers [26]. While obligate anaerobes belonging to classes such as Clostridia and Bacteroidetes appear to play pivotal roles in the prevention of dysbiosis, the population of facultative anaerobes, including pathogenic Enterobacteriaceae, increases when that of obligate anaerobes is reduced [11]. Indeed, the present study demonstrated that endotoxemia evoked the decrease in the populations of bacteria belonging to the Bacteroidetes classe in the colon, while the exact reason for this change remains to be elucidated. In lower-level analyses of the gut microbiota, endotoxemia upregulated the Clostridium perfringens while decreasing the Bacteroides fragilis subgroups irrespective of hyperglycemic condition. It should be noted that hyperglycemia per se evoked alterations of the gut microbiota of the colon even in lower-level analysis, e.g., decrease of the Lactobacillus reuteri subgroup and increase of Staphylococci in the healthy condition, and decrease of Enterococcus and Enterobacteriaceae in the endotoxemic condition. While we did not evaluate how these alterations in the colon microbiota were induced, it is plausible to consider that these changes could affect the gut immune function.

Although we demonstrated that inflammatory mediators and differentiation of lymphocytes in MNLs, and microbiological constitute in the intestine were modulated by persistent hyperglycemia and endotoxin in this study independently or dependently, it is mandatory to discuss how these changes interact with each other and consider clinically relevant implications of this study. Hyperglycemia evoked inflammatory cytokines while promoting differentiation to Th2 and Treg which are responsible for the release of anti-inflammatory cytokines in MNLs. Although these changes seem to be contradictory, it is often the case that hyper-inflammatory and anti-inflammatory responses coexist in the disease condition such as sepsis in which hyper-inflammatory condition is followed by anti-inflammatory response at the almost same time [27]. Thus, it is likely that the alteration of immune status induced by hyperglycemia contributed to the deterioration of the gut immune function, leading to the alteration of the gut microbiota. On the other hand, endotoxemia evoked the alteration of the gut microbiota without the effects on inflammatory cytokines and $\mathrm{CD} 4^{+} \mathrm{T}$ cells differentiation in MNLs. Although the true mechanism was not elucidated in this study, some possibilities should be considered. One $\mathrm{mg} /$ $\mathrm{kg}$ LPS, which was less than the dose of $4 \mathrm{mg} / \mathrm{kg}$ we used in the previous study [14], might not be enough to evoke the alteration of cytokine production and $\mathrm{CD} 4^{+} \mathrm{T}$ cells differentiation, even though the survival rate of the EH group at 24 hours was about $80 \%$ in our preliminary study, which implies the severity of disease condition induced by this dose of LPS. Otherwise, concentration of cytokine had already peaked out and the alteration of $\mathrm{CD}^{+} \mathrm{T}$ cells differentiation had already finished at 24 hours. Further study is warranted to elucidate the mechanism how these changes interact and modulate the gut immune function. While the true mechanism was not elucidated in this study, the alteration of the gut microbiota, which was induced by hyperglycemia and endotoxemia in this study, could evoke the deteriorated gut immune function and bacterial translocation into systemic circulation [28]. Considering the results of this study, an intervention should be taken into account to maintain the homeostasis of the microbiota and prevent the gut immune dysfunction in patients with uncontrolled hyperglycemia and endotoxemia, such as administration of probiotics. Previous study demonstrated that the Lactobacillus reuteri subgroup considered as one of probiotic bacterial strains, which was reduced by hyperglycemia in this study, may be able to depress growth, adherence and virulence of Staphylococci $[29,30]$. It is expected that new interventions are developed to maintain the intestinal microbiota in critically ill patients in future studies.

Some limitations of this study must be borne in mind while interpreting the data herein. First, some may argue that we should have measured the respective protein concentrations directly caused by the alterations of cytokine mRNA. Although previous study showed that protein level was remarkably elevated as well as cytokine mRNA in an endotoxemic model [31], the protein concentrations are not necessarily dependent on the changes of cytokine mRNA. Another issue is the semiquantitative strategy applied may provide only a limited view of the ecological disruption potentially caused by endotoxemia. Since the gut microbiota varies widely in composition among individuals even in health depending on their geographical origin and/or location within the gut, further examination of intestinal environments, such as of organic acids, may be required to determine 
the abnormal changes of the microbiota. Second, persistent hyperglycemia observed in critically ill patients is basically not caused by continuous glucose administration but by endogenous alterations such as resistance to insulin [2]. However, we used exogeneous glucose infusion model to mimic clinical situations since it is difficult to maintain stable hyperglycemia level in rodent models without exogeneous glucose, and exogeneous glucose infusion, such as parenteral nutrition, was often performed in the intensive care unit, while LPS model did not introduce hyperglycemia in this study. Furthermore, there are a large number of confounding variables in clinical settings, such as antibiotic use, starvation, enteral feeding and others which trigger alterations of the gut microbiota. Ultimately, the effects of oral antibiotics or probiotics on gene expression in the MLNs should be settled by further study. After all, in addition to having different microbiota from humans, it may not be possible to precisely mimic clinical situations in rodent models under experimental conditions. Third, the values of $\mathrm{CD} 4^{+} \mathrm{T}$ cells, evaluated by the change of transcriptional factors through RT-PCR, could not reflect the actual number of each lymphocyte since extraction of m-RNA was performed from the contents of MNLs without cell sorting by FACS, and the alteration of transcriptional factors could not always be consistent with the number of lymphocytes. Although these values of $\mathrm{CD} 4^{+} \mathrm{T}$ cells could not represent the actual number, the trend of $\mathrm{CD}_{4}^{+} \mathrm{T}$ cells differentiations could be detected by this measurement method. Finally, we performed lots of multiple comparisons to evaluate the alteration of bacteria in colon microbiota, which might lead to the false positive. However, the possibility of false positive could be a minimum, since we performed multiple comparisons after detection of interaction between two factors, hyperglycemia and endotoxemia.

\section{Conclusions}

In conclusion, the present study showed that persistent hyperglycemia evoked by exogenous glucose infusion for 24 hours modulates both inflammatory responses and $\mathrm{CD}^{+} \mathrm{T}$ cells differentiation in the MLNs, and induces the alterations of the colon microbiota. Further study is warranted to elucidate the causal relationship between persistent hyperglycemia and altered gut immunity as well as clinical implications of these changes in gut.

\section{Additional file}

Additional file 1: Figure $\mathbf{S 1}$ The classification of bacteria in the colon microbiota. This figure represents the classification of colon bacteria which was evaluated in this study. (TIFF $86 \mathrm{~kb}$ )

\section{Competing interests}

The authors declare that they have no competing interests.

\section{Authors' contributions}

KM carried out the whole study procedure, collected data, and drafted the manuscript. TS participated in the design of the study, and helped in carrying out the statistical analysis and drafting the manuscript. TI helped to perform the PCR analysis. KI helped to carry out the study procedure. TA and KN helped to perform the microbiota analyses. HS advised for designing the study protocol, and helped to draft the manuscript. TY participated in the design of the study, and advised for the execution of the study procedure. SM gave the technical support in carrying out the study procedure. SK gave advice for the study protocol, and helped to draft the manuscript. NK participated in the design of the study, and coordinated the whole study procedure. HM coordinated the study protocol, and helped to draft the manuscript. All authors read and approved the final manuscript.

\section{Acknowledgments}

We wish to express our sincere gratitude to Mr. Norikatsu Yuki and Mr. Akira Takahashi of the Yakult Central Institute for Microbiological Research for their valuable assistance in performing the microbiota analyses. Furthermore, we would like to give special thanks to Dr Takayuki Abe who gave us advice as a statistician with respect to the statistical analyses.

\section{Author details}

${ }^{1}$ Department of Anesthesiology, Keio University School of Medicine, 35 Shinanomachi, Shinjuku-ku, Tokyo 160-8582, Japan. ${ }^{2}$ Yakult Central Institute for Microbiological Research, 5-11 Izumi, Kunitachi-shi, Tokyo 186-0012, Japan.

Received: 6 December 2014 Accepted: 8 July 2015

Published online: 23 July 2015

\section{References}

1. Falciglia M, Freyberg RW, Almenoff PL, D'Alessio DA. Hyperglycemia-related mortality in critically ill patients varies with admission diagnosis. Crit Care Med. 2009;37:3001-9.

2. Turina M, Fry DE, Polk Jr HC. Acute hyperglycemia and the innate immune system: clinical, cellular, and molecular aspects. Crit Care Med. 2005;33:1624-33.

3. van den Berghe $G$, Wouters P, Weekers F, Verwaest C, Bruyninckx F, Schetz $\mathrm{M}$, et al. Intensive insulin therapy in critically ill patients. N Engl J Med. 2001;345:1359-67.

4. Sakowicz-Burkiewicz M, Grden M, Maciejewska I, Szutowicz A, Pawelczyk T. High glucose impairs ATP formation on the surface of human peripheral blood B lymphocytes. Int J Biochem Cell Biol. 2013;45:1246-54.

5. Gonzalez Y, Herrera MT, Soldevila G, Garcia-Garcia L, Fabián G, PérezArmendariz EM, et al. High glucose concentrations induce TNF-a production through the down-regulation of CD33 in primary human monocytes. BMC Immunol. 2012;13:19.

6. Esposito K, Nappo F, Marfella R, Giugliano G, Giugliano F, Ciotola M, et al. Inflammatory cytokine concentrations are acutely increased by hyperglycemia in humans: role of oxidative stress. Circulation. 2002;106:2067-72.

7. Koboziev I, Karlsson F, Grisham MB. Gut-associated lymphoid tissue, T cell trafficking, and chronic intestinal inflammation. Ann N Y Acad Sci. 2010;1207 Suppl 1:E86-93.

8. Abraham C, Cho JH. IL-23 and autoimmunity: new insights into the pathogenesis of inflammatory bowel disease. Annu Rev Med. 2009;60:97110.

9. Tamion F, Richard V, Lyoumi S, Daveau M, Bonmarchand G, Leroy J, et al Gut ischemia and mesenteric synthesis of inflammatory cytokines after hemorrhagic or endotoxic shock. Am J Physiol. 1997;273(2 Pt 1):G314-321.

10. Macpherson AJ, Harris NL. Interactions between commensal intestinal bacteria and the immune system. Nat Rev Immunol. 2004;4:478-85.

11. Winter SE, Lopez CA, Bäumler AJ. The dynamics of gut-associated microbia communities during inflammation. EMBO Rep. 2013;14:319-27.

12. Shimizu K, Ogura H, Hamasaki T, Goto M, Tasaki O, Asahara T, et al. Altered gut flora are associated with septic complications and death in critically ill patients with systemic inflammatory response syndrome. Dig Dis Sci. 2011;56:1171-7. 
13. Yajima S, Morisaki H, Serita R, Suzuki T, Katori N, Asahara T, et al. Tumor necrosis factor-alpha mediates hyperglycemia-augmented gut barrier dysfunction in endotoxemia. Crit Care Med. 2009;37:1024-30.

14. Matsuda K, Tsuji H, Asahara T, Matsumoto K, Takada T, Nomoto K. Establishment of an analytical system for the human fecal microbiota, based on reverse transcription-quantitative PCR targeting of multicopy rRNA molecules. Appl Environ Microbiol. 2009;75:1961-9.

15. Jansen GJ, Wildeboer-Veloo AC, Tonk RH, Franks AH, Welling GW. Development and validation of an automated, microscopy-based method for enumeration of groups of intestinal bacteria. J Microbiol Methods. 1999:37:215-21.

16. Tamoutounour S, Henri S, Lelouard H, de Bovis B, de Haar C, van der Woude CJ, et al. CD64 distinguishes macrophages from dendritic cells in the gut and reveals the Th1-inducing role of mesenteric lymph node macrophages during colitis. Eur J Immunol. 2012;42:3150-66.

17. Cavaillon JM, Adib-Conquy M. Monocytes/macrophages and sepsis. Crit Care Med. 2005:33 Suppl 12:S506-509.

18. Wen Y, Gu J, Li SL, Natarajan R, Nadler JL. Elevated glucose and diabetes promote interleukin-12 cytokine gene expression in mouse macrophages. Endocrinology. 2006;147:2518-25.

19. Wynn $T A \cdot T(H)-17$ : a giant step from $T(H) 1$ and $T(H) 2$. Nat Immunol. 2005:6:1069-70

20. Jiang LN, Yao YM, Sheng ZY. The role of regulatory T cells in the pathogenesis of sepsis and its clinical implication. J Interferon Cytokine Res. 2012;32:341-9

21. Hotchkiss RS, Karl IE. The pathophysiology and treatment of sepsis. N Engl J Med. 2003;348:138-50

22. Monneret G, Debard AL, Venet F, Bohe J, Hequet O, Bienvenu J, et al. Marked elevation of human circulating $\mathrm{CD} 4^{+} \mathrm{CD} 25^{+}$regulatory $\mathrm{T}$ cells in sepsis-induced immunoparalysis. Crit Care Med. 2003;31:2068-71.

23. Viardot A, Grey ST, Mackay F, Chisholm D. Potential antiinflammatory role of insulin via the preferential polarization of effector T cells toward a T helper 2 phenotype. Endocrinology. 2007;148:346-53.

24. Tiittanen M, Huupponen JT, Knip M, Vaarala O. Insulin treatment in patients with type 1 diabetes induces upregulation of regulatory T-cell markers in peripheral blood mononuclear cells stimulated with insulin in vitro. Diabetes. 2006:55:3446-54

25. Härdtner C, Mörke C, Walther R, Wolke C, Lendeckel U. High glucose activates the alternative ACE2/Ang-(1-7)/Mas and APN/Ang IV/IRAP RAS axes in pancreatic $\beta$-cells. Int J Mol Med. 2013;32:795-804.

26. Lawley TD, Walker AW. Intestinal colonization resistance. Immunology. 2013:138:1-11.

27. Angus DC, van der Poll T. Severe sepsis and septic shock. N Engl J Med. 2013:369:840-51

28. Choudhry MA, Rana SN, Kavanaugh MJ, Kovacs EJ, Gamelli RL, Sayeed MM Impaired intestinal immunity and barrier function: a cause for enhanced bacterial translocation in alcohol intoxication and burn injury. Alcohol. 2004:33:199-208

29. Li J, Wang W, Xu SX, Magarvey NA, McCormick JK. Lactobacillus reuteriproduced cyclic dipeptides quench agr-mediated expression of toxic shock syndrome toxin-1 in staphylococci. Proc Natl Acad Sci U S A. 2011;108:3360-5.

30. Prince T, McBain AJ, O'Neill CA. Lactobacillus reuteri protects epidermal keratinocytes from Staphylococcus aureus-induced cell death by competitive exclusion. Appl Environ Microbiol. 2012;78:5119-26.

31. DeForge LE, Remick DG. Kinetics of the IL-6 and IL-8 gene expression in LPS-stimulated human whole blood. Biochem Biophys Res Commun. 1991;174:18-24.

\section{Submit your next manuscript to BioMed Central and take full advantage of:}

- Convenient online submission

- Thorough peer review

- No space constraints or color figure charges

- Immediate publication on acceptance

- Inclusion in PubMed, CAS, Scopus and Google Scholar

- Research which is freely available for redistribution 